\title{
Configurações
}

Revista de sociologia

\section{Vieillissement, relations intergénérationnelles et récession. La crise financière aura-t-elle raison de la révolution des âges?}

Ageing,intergenerational relations and recession. Can the financial crisis take over the revolution of the age-groups?

\section{Michel Loriaux}

\section{OpenEdition}

\section{Journals}

Édition électronique

URL : http://journals.openedition.org/configuracoes/272

DOI : 10.4000/configuracoes.272

ISSN : 2182-7419

Éditeur

Centro de Investigação em Ciências Sociais

Édition imprimée

Date de publication : 1 janvier 2008

Pagination : 11-25

ISSN : 1646-5075

Référence électronique

Michel Loriaux, «Vieillissement, relations intergénérationnelles et récession. La crise financière aura-telle raison de la révolution des âges? », Configurações [En ligne], 4 | 2008, mis en ligne le 12 février 2012, consulté le 01 mai 2019. URL : http://journals.openedition.org/configuracoes/272 ; DOI : $10.4000 /$ configuracoes. 272

Ce document a été généré automatiquement le 1 mai 2019.

(c) CICS 


\title{
Vieillissement, relations
} intergénérationnelles et récession. La crise financière aura-t-elle raison de la révolution des âges?

\author{
Ageing,intergenerational relations and recession. Can the financial crisis take \\ over the revolution of the age-groups?
}

\author{
Michel Loriaux
}

\section{L'invention du « vieillissement»}

1 Le terme de révolution est souvent galvaudé ou dénaturé dans la mesure où on tend à l'utiliser pour désigner une simple évolution d'un des grands paramètres sociétaux (religiosité, criminalité, urbanisme, etc.). En réalité, il serait plus correct de réserver l'expression à des mutations profondes de nos sociétés, comme il s'en est produit un certain nombre au cours du vingtième siècle, et plus particulièrement ces dernières décennies. La révolution des âges fait partie de ces mutations et constitue sans aucun doute un des courants lourds les plus significatifs de notre histoire récente, au même titre que la féminisation de l'emploi ou la révolution de la communication.

2 Les transformations avaient pourtant commencé beaucoup plus tôt, voici un siècle ou un siècle et demi, lorsque la natalité a amorcé son grand déclin historique à la fin de l'ancien régime et au début de la phase d'industrialisation de nos sociétés occidentales. A l'origine, le phénomène a surtout été perçu dans sa dimension exclusivement démographique de dénatalité et de dépopulation, des maux redoutés à une époque où le nombre des hommes constituait un des facteurs essentiels de la richesse des nations. Moins de bras dans l'agriculture, moins d'hommes de troupe à lever contre d'hypo- thétiques ennemis, moins de prolétaires pour répondre à la demande croissante des manufactures et des industries 
gourmandes en main-d'œuvre, moins de consommateurs potentiels : les raisons étaient nombreuses de redouter une raréfaction des naissances, même si la tendance était soutenue par le déclin parallèle de la mortalité infantile qui limitait l'ampleur des coupes sombres à la base de la pyramide des âges.

Il fallut plusieurs décennies pour comprendre que ce qui se passait du côté de la base et des âges jeunes avait nécessairement une influence sur le sommet et les âges élevés. Non pas forcément que les vieux augmentaient en nombre, comme s'il s'était agi de générations spontanées, mais parce que toute réduction à la base de la pyramide modifiait le poids relatif des ânés qui prenaient lentement une importance proportionnelle accrue.

4 C'est apparemment au démographe français Alfred Sauvy, que revint le mérite d'avoir identifié pour la première fois ce phénomène ou, en tout cas, de l'avoir nommé puisqu'on lui attribue la paternité de l'expression "vieillissement démographique » apparue dans la littérature scientifique en 1929. A partir de ce moment, le duo « dénatalité-dépopulation » se transforma en un trio " dénatalité-dépopulation-vieillissement » qui comportait tous les germes « maléfiques » des inquiétudes collectives à venir.

5 Car A. Sauvy n'avait probablement pas utilisé innocemment le mot "vieillisse- ment » pour décrire ce qui n'était en définitive qu'un simple changement de structure de population. Tout porte à croire que l'analogie avec le vieillissement individuel et biologique était voulue, puisqu'elle permettait de transférer les heurs et malheurs de ce processus de sénescence individuelle au corps social tout entier. Ce qui n'inspirait que souffrances et perspectives de mort à tout être humain devait nécessairement jeter dans l'effroi les sociétés menacées de vieillissement démographique afin de susciter un réflexe salutaire de redressement à travers une relance de la fécondité et le repeuplement des berceaux abandonnés. Bref, il fallait faire peur et le résultat fut atteint au-delà de toute espérance.

\section{Dénoncer le mal sénile}

6 On ne compte plus les théories et les arguments qui furent développés par les contempteurs du vieillissement pour démontrer qu'une population vieillissante était menacée dans ses fonctions vitales : perte de dynamisme économique et de compétitivité commerciale, réduction de la consommation des ménages et désintérêt pour les investissements productifs à long terme, dépendance migratoire accrue vis-à-vis de l'étranger et risque de submergement du Nord par le Sud, diminution de la flexibilité et de la mobilité de la main-d'œuvre, élévation du coût du travail et diminution de sa compétitivité face au capital, accentuation du conservatisme politique et du risque de prise de pouvoir gérontocratique, augmentation du chômage des jeunes et développement de la xéno- phobie, perte de cohésion sociale et réduction des solidarités intergénérationnelles, etc.

7 Parfois, un argument asséné comme un coup de massue résumait tous les autres : il n’y a pas dans l'histoire des sociétés d'exemples qui infirmeraient l'hypothèse que la croissance économique va toujours de pair avec la croissance démographique. Et sur- tout ne croyez pas qu'il s'agisse là d'un vieux slogan démodé. On en trouve par exemple la trace assez récemment aussi bien dans un ouvrage d'Alain Minc datant de $1987^{1}$ que dans le Livre Vert de la Commission européenne (2005) sur les relations intergénérationnelles². 
8 L'idée n'est pas ici de contester massivement la vérité de chacun de ces arguments, mais plutôt de faire remarquer que des relations qui ont pu être vraies dans un contexte sociétal donné peuvent cesser de l'être dans un autre. C'est d'ailleurs un des principaux écueils auxquels les sciences sociales sont confrontées depuis toujours, à savoir, n'être jamais assurées d'aucune constance dans le temps et/ou dans l'espace des paramètres sociétaux ni des prétendues " lois » les régissant dans un monde en évolution permanente, fait de perpétuels mouvements de décomposition/recomposition. Là où les sciences exactes peuvent se prévaloir de la condition du « toutes autres choses égales par ailleurs ", les sciences sociales doivent reconnaitre que la seule constante qui s'impose à elles est plutôt celle du « toutes autres choses inégales par ailleurs ", qui transforme les lois universelles en simples relations imparfaites de covariation statistique ou de cooccurrence.

Par exemple, même si on peut concéder aux vilipendeurs du vieillissement démographique qu'à l'origine du phénomène, il y avait probablement des raisons de redouter une pénurie de population active, dans des sociétés encore profondément rurales et qui reposaient largement sur un facteur travail dominant dans le processus de production industrielle, aujourd'hui, il serait absurde de ne pas reconnaître que le monde a profondément changé de nature et que le travail est en perte de vitesse face à la concentration capitalistique : l'emploi stagne ou régresse, comme les salaires, et il se précarise avec les temps partiels, les contrats à durée déterminée, les emplois intérimaires, tandis que le chômage ne cesse de progresser, surtout dans les catégories fragilisées, en particulier les jeunes et les travailleurs âgés, mais aussi les moins qualifiés.

10 Dans de telles conditions est-il encore raisonnable de prétendre que nos populations s'étiolent et que l'offre de travail est insuffisante, sauf dans quelques secteurs bien déterminés où souvent ce sont d'ailleurs nos dirigeants eux-mêmes qui ont organisé la pénurie par des mesures inappropriées (cfr le numérus clausus en médecine)?

\section{La mortalité en première ligne}

11 Mais une illustration plus significative encore peut être donnée à propos du vieillissement lui-même. Car ce n'est pas seulement la société qui s'est transformée, c'est aussi le vieillissement qui a changé d'intensité et surtout de nature. Pour saisir l'importance de ces mutations, il faut à nouveau se reporter à l'origine du phénomène. Dans les premières décennies de manifestation du vieillissement démographique, il fut clairement établi que sa cause unique était la diminution de la fécondité, et en aucune manière la baisse de la mortalité, puisque celle-ci ne profitait qu'aux enfants en bas âges et constituait donc plutôt un facteur de rajeunissement de la population (ou du moins de moindre vieillissement ${ }^{3}$.

12 Cette interprétation fut correcte au moins jusqu'à la seconde moitié du XXème siècle, époque où la lutte contre la mortalité prolongea ses effets favorables jusqu'aux classes âgées et très âgées et où les gains en faveur des enfants commencèrent à ralentir au fur et à mesure où une sorte de niveau plancher très bas était approché.

13 A partir de ce moment, le discours des démographes dut s'adapter pour reconnaitre que le recul de la mortalité était devenu aussi une cause potentielle de vieillisse- ment, même si elle n'intervenait alors qu'en deuxième position derrière le déclin de la fécondité. Encore faut-il rappeler que durant l'immédiat après guerre et pendant pratiquement 
deux décennies, le fameux baby-boom put créer l'illusion que la bataille du vieillissement avait été gagnée, ou était sur le point de l'être, puisque la fécondité avait amorcé une spectaculaire relance jusqu'au moment où un retournement inattendu se produisit, autour des années 1965, et où la fécondité repris sa course historique à la baisse qui conduisit aux niveaux enregistrés de nos jours, pratiquement tous inférieurs au seuil de remplacement des générations (2,1 enfants/femme) et parfois proche de l'enfant unique, comme dans les pays méditerranéens.

Aujourd'hui, la cause est entendue: les progrès constants de l'espérance de vie, et principalement de l'espérance de vie aux âges élevés (60 ans et plus, par exemple) ne laissent plus planer aucun doute sur le fait que le recul de la mortalité est devenu une cause essentielle du vieillissement.

Beaucoup d'experts s'accordent même pour dire que les changements dans les régimes de mortalité sont devenus la cause principale du phénomène, supplantant la grande cause historique qu'était le déclin de la fécondité, au point que si celle-ci reprenait durablement vigueur, il n'y aurait aucune chance que le vieillissement régresse pour autant, tout au plus serait-il appelé à progresser moins rapidement. Mais pour l'heure, les deux causes étant simultanément présentes, le vieillissement poursuit sa progression rapide, à la fois par la base de la pyramide (moins d'enfants, aussi bien en valeur absolue que relative) et par le sommet (plus de vieux qui vivent de plus en plus longtemps). Cette situation explique notamment le fait que les deux courbes des moins de 20 ans et des plus de 60 ans se sont déjà croisées en ciseaux dans la plupart des pays aux environ de l'année 2005 et qu'elles ne cessent de s'éloigner l'une de l'autre dans les perspectives à l'horizon 2050.

\section{Une révolution aussi profonde que silencieuse}

16 Là est sans doute le véritable sens de la révolution des âges. Jamais dans l'histoire des populations humaines, les jeunes n'avaient été moins nombreux que les vieux. Au contraire, pendant de longs siècles, les vieux, au sens des sexagénaires et au-delà, ne représentaient qu'une petite minorité des populations, par exemple moins de $5 \%$, et même lorsque le vieillissement a commencé à s'installer, il fallut longtemps avant qu'ils atteignent un dixième de l'effectif total, ce qui est loin de la situation actuelle.

Dans un certain sens, on peut dire que le vieillissement ne devrait plus être la préoccupation principale, dans la mesure où la montée en puissance des ainés, pour interpellante qu'elle soit, n'est plus en soi la source majeure d'inquiétude. Certes, des questions comme celle de l'hébergement des personnes âgées dépendantes, ou comme la progression des dépenses de santé avec l'avancée en âge, peuvent se poser avec une certaine acuité. Mais la question dominante est devenue celle des relations entre les générations. En somme, les évolutions démographiques depuis 150 ou 200 ans ont d'abord mis en évidence la dénatalité, puis le vieillissement et enfin la multiplicité générationnelle. Car c'est bien cette multiplicité et cette diversité générationnelles qui sont devenues la source de grands enjeux sociétaux. A cause des changements de profils pyramidaux, les relations entre les générations ont été bouleversées. Et ce d'autant plus qu'en même temps les générations ont profondément changé de visages.

18 Aux mêmes âges, les jeunes adultes et les vieux, pour n'évoquer que de grandes catégories schématiques, n'ont plus aujourd'hui les caractéristiques physiques, sociales ou culturelles qu'ils avaient hier, et probablement encore moins celles qu'ils auront demain. 
En gros, les citoyens ont acquis, au fil des ans, des propriétés qui les distinguent profondément de leurs parents et grands parent : meilleur régime alimentaire et en général meilleures conditions de vie, meilleure scolarisation et meilleure formation professionnelle, emplois moins pénibles et moins dangereux, carrières linéaires et rémunérations supérieures, accession plus rapide à la propriété et acquisition de patrimoines importants, etc. Mais au-delà de ces attributs matériels changeants, ce sont aussi les valeurs et les modèles culturels qui ont rapidement évolué, ce que les historiens appel- lent parfois l'accélération de l'histoire, un phénomène qui tend à brouiller toutes les cartes et à créer dans la population un profond sentiment d'anomie et d'insécurité.

19 Non seulement les diverses générations ont des valeurs, des aspirations, des besoins différents en fonction de leurs âges et des époques auxquelles elles ont été socialisées ou des événements qu'elles ont vécu, mais de surcroît ces valeurs, ces aspirations et ces besoins ont souvent subi des mutations plus ou moins profondes au cours de leur avancée vitale. Le résultat est une société hétérogène dans laquelle les valeurs collectives semblent dissoutes dans le magma des valeurs individuelles, aucune dominante ne paressant devoir s'imposer, en dehors bien sûr de l'individualisme et de l'ultralibéralisme dominant.

\section{Et la crise survint ...}

20 Or, c'est précisément à ce moment crucial pour l'avenir des sociétés vieillissantes que survient une crise financière et économique d'une ampleur inégalée dont tout le monde perçoit instinctivement qu'elle va avoir des incidences énormes sur ces mêmes sociétés.

21 La question posée ici est de savoir en quoi l'évolution du vieillissement et des relations intergénérationnelles seront affectées par cette crise (et réciproquement). Sans doute estil toujours dangereux de s'aventurer dans des perspectives minées par une incertitude majeure, mais en même temps, les sciences sociales ont pour mission d'être des éclaireurs du futur et de tenter d'anticiper des évolutions, fusse parfois seulement pour les éviter. En ce qui concerne la crise financière de l'automne 2008, des conséquences sont d'ores et déjà connues, d'autres sont plus incertaines et dépendent des réponses étatiques qui lui seront données.

22 Parmi les premières, on peut naturellement citer la perte du pouvoir d'achat qui va affecter les couches de la population dont les revenus dépendent principalement de l'épargne, présente ou antérieure, et particulièrement les retraités. Au sein de cette catégorie sociale, nombreux sont ceux qui avaient constitué une épargne de prévoyance pour sécuriser leurs vieux jours, selon l'expression consacrée, et pour compléter une retraite légale qu'ils savaient insuffisante pour leur éviter une diminution de niveau de vie. Une partie d'entre eux, qui ont joué la prudence en préférant les taux faiblement rémunérateurs des carnets d'épargne aux attraits des rendements élevés promis par les fonds de placement ou les montages financiers sophistiqués, verront peut-être leurs revenus partiellement préservés, si du moins ils n'ont pas choisi la mauvaise banque insolvable et si les états-nations respectent leurs engagements de couvrir les placements « sains » jusqu'à des montants assez confortables.

23 Mais pour les autres qui ont misé sur des produits dits aujourd'hui « toxiques », qui leurs avaient pourtant été chaudement recommandés par leurs conseillers bancaires, la déconvenue risque d'être forte, en découvrant brutalement que leurs avoirs ont fondu 
comme neige au soleil et que les pertes s'élèvent à $50 \%, 80 \%$ ou $99 \%$ des valeurs nominales de leurs titres. Tous n'étaient pourtant pas des spéculateurs et leur seul tort a souvent été de faire confiance à des banquiers et des assureurs cupides ou séduits euxmêmes par les sirènes du profit facile. Comme la crise financière est loin d'être terminée, on évalue encore mal son impact réel sur les populations, beaucoup d'épargnants ayant la pudeur ou la honte de dévoiler leurs pertes, mais il est certain que les conséquences seront plus dommageables pour les individus que pour les institutions financières, au secours desquelles les états pompiers sont rapidement intervenus pour éteindre les incendies qui menaçaient partout. Il y aura certes des fusions, des rachats, des nationalisations, mais au bout du compte le système reviendra à un certain équilibre parce que le capitalisme ne peut survivre sans ses organes de transmission et d'amplification des flux financiers. Le seul espoir nourri par certains observateurs, peutêtre un peu trop pétris de bons sentiments et de rêves utopiques est qu'une meilleure régulation et un meilleur contrôle international sera organisé pour limiter les risque de nouveau dérapage, comme ce fut le cas dans les années qui suivirent la grande crise de 1929, notamment avec la mise en place des institutions de Bretton Wood.

\section{Des dégâts inestimables}

Mais même si cet espoir se réalise, les dégâts collatéraux auront été importants et ne seront pas réparables avant longtemps. Des retraités qui croyaient s'être mis à l'abri des aléas de l'existence auront été plongés dans la pauvreté et l'assistanat, incapables de pourvoir à certains de leurs besoins essentiels, comme les soins médicaux et même peutêtre l'alimentation de base. Des maux, comme la famine ou certaines formes de morbidité et de précarité qui avaient été fortement réduits, sinon éradiqués, à la fin du XXème siècle grâce à des politiques de protection sociale assez " généreuses " feront leur réapparition massive et donneront à nouveau l'occasion à la charité privée de s'exercer, comme c'était le cas au XIXème siècle dans les souffrances de l'accouchement des sociétés industrielles et de la modernité. A la limite, les progrès de longévité, qui semblaient devoir perdurer longtemps encore, s'arrêteront et donneront raison à ceux qui annonçaient récemment un renversement de tendance dans les prochaines décennies : la crise ne fera d'attiser des risques (sociaux, écologiques, etc.) qui paraissaient pouvoir être maîtrisés.

Même les pensionnés qui avaient fait confiance aux systèmes de retraite par répartition et qui n'avaient pas pensé devoir céder aux sollicitations des vendeurs d'épargnepension et de troisième pilier, auront le sentiment d'avoir été trompés lorsqu'ils découvriront que le niveau des retraites publiques stagne, malgré le renchérissement du coût de la vie, liée aux pénuries (organisées ou non) et même régresse par rapport aux salaires parce que l'obligation de faire face aux engagements pris par des états à l'égard du système financier alourdira la dette publique et rendra impossible toute réévalution des retraites.

Mais les retraités ne seront pas les seules victimes de ces désordres financiers, les jeunes et les classes d'âges intermédiaires (les " adultes ») seront également soumis aux contraintes d'une économie qui aura du mal à absorber le contrecoup d'une crise financière qui se sera transformée en crise économique mais aussi en crise de confiance et de solidarité. Le chômage des jeunes augmentera au-delà des situations actuelles déjà fortement dégradées et les plans sociaux fleuriront pour éliminer les travailleurs âgés, avec un regain des régimes de prépensionnement, malgré la tendance affichée depuis 
quelques années par les pouvoirs publics à allonger les carrières et à relever l'âge de départ à la retraite (réel et /ou légal).

Parallèlement, les salaires resteront bloqués et les emplois précaires se multiplieront de sorte que la répartition du revenu national entre le travail et le capital continuera à se dégrader en faveur du capital. Les actifs qui avaient choisi de créer leur propre entreprise auront beaucoup de difficultés à obtenir des banques les ouvertures de crédit nécessaires et un nombre grandissant de patrons de PME seront acculés à la faillite, à laquelle d'ailleurs même les grands groupes internationaux (dans des secteurs comme l'automobile, la métallurgie, la pétrochimie, etc.) seront confrontés. Dans des cas extrêmes, ce seront parfois les états eux-mêmes qui seront mis dans l'impossibilité d'honorer leurs engagements, aussi bien internes (le paiement des fonctionnaires, le versement des retraites, etc.) qu'internationaux (le remboursement de ses emprunts, le paiement de leurs achats, etc.).

Or, tout cela ne relève pas d'un scénario de science-fiction catastrophiste puis- qu'on connaît déjà des exemples de toutes ces situations. La menace est réelle et déjà présente. Elle ne pourra que s'amplifier si une réforme totale de tout notre système de fonctionnement sociétal n'est pas mise en œuvre rapidement. Au-delà des banques à encadrer et des parachutes dorés à limiter et à réguler, de la pauvreté à éradiquer (selon les objectifs du Millénaire pour le Développement - les OMD des Nations Unies), c'est toute la question de la lutte contre les inégalités et celle du partage équitable du produit collectif mondial entre les pays, les classes sociales et les générations qui devra être débattue en profondeur en renonçant à l'idéologie castratrice de l'ultra-libéralisme qui a été tellement dévastatrice, mais qui semblait être tellement la seule voie possible qu'elle a fini par enlever toute capacité de discernement et de libre conscience aux experts et aux décideurs du monde entier.

29 Aujourd'hui que la mystification a été dénoncée par les événements, le temps est peutêtre venu, non pas seulement de sauver le système financier international, mais avant tout de réfléchir aux moyens de repositionner l'Homme au cœur de la société, et plus spécialement de l'économie qui s'était barricadée dans ses forteresses en apparence inexpugnables. " A quelque chose, malheur est bon » dit le proverbe, et on se prend à espérer que derrière tous les malheurs qui se profilent, se produira un sursaut salutaire pour implanter un modèle de développement plus humain, pas seulement au Sud, mais également au Nord.

\section{Un prospectiviste mal inspiré ?}

Il y a maintenant plus de 20 ans, un essayiste français, proche des milieux de la finance et conseiller économique de grandes entreprises, avait défendu une thèse provocatrice selon laquelle plus une société manifestait des velléités égalitaires, plus elle risquait de succomber à des effets pervers qui aboutissaient in fine à générer plus d'inégalités. Dans un des chapitres de sa " Machine égalitaire » (1987), Alain Minc, stigmatisait particulièrement le vieillissement démographique auquel il reprochait toutes les dérives collectives en attribuant aux sociétés vieillissantes la caractéristique d'être moins productives et donc de rendre la redistribution impossible dans un contexte où

31 " les besoins augmentent au moment où les ressources diminuent ". Il est vrai qu'à l'époque, les « trente glorieuses » étaient déjà loin en arrière et que les crises pétrolières 
avaient frappé de plein fouet les sociétés industrielles justifiant une certaine vague de pessimisme qui s'était répandue au sein des médias et des élites.

Le portrait dressé par l'auteur était réellement apocalyptique : en cause d'abord le coût grandissant des soins de santé, et des assurances maladie, car si « l'évolution des mœurs médicalise la société, son vieillissement la surmédicalise ». Il en résultera un accroissement des inégalités entre les catégories de retraités, les plus démunis devront passer sous les fourches caudines du rationnement hospitalier tandis que les plus nantis

" grâce à des assurances souscrites durant leur vie active par eux-mêmes ou par leurs entreprises, pourront avoir accès aux traitements les plus onéreux ».

Du côté des retraites, la situation ne sera pas moins critique et Alain Minc affirme que les systèmes courent à la catastrophe et sont menacés d'explosion à cause de la dégradation rapide du rapport actifs/inactifs avec des générations issues du baby boom qui seront à la retraite entre 2010 et 2025 et qui pèseront lourd en face des classes creuses de l'après 1965. Le système de financement des retraites par répartition ne pourra résister à une expansion économique ralentie et à une démographie déliquescente et même si la capitalisation sera appelée à la rescousse pour venir compléter la répartition, elle ne suffira pas à annuler la pression de sorte que de nouvelles inégalités se développeront inévitablement.

Même les assurances familiale et chômage ne tireront pas réellement profit de l'allègement des charges dans ces secteurs, du fait d'une fécondité moindre et d'une pression réduite sur l'emploi, " parce que le ralentissement de l'économie inhérent au vieillissement des populations réduira encore le volume de l'emploi disponible et jouera en sens inverse des tendances démographiques. Les équipements collectifs liés à l'enfance (maternités, crèches, école) seront sous-utilisés et bientôt fermés, de sorte que leur coût augmentera en même temps que le déficit de l'Etat. Mais la demande globale sera elleaussi atteinte dans la mesure où une population vieillie consomme moins, sacrifie peu à des investissements à long terme, tel le logement, et accorde d'avantage de prix au présent qu'à l'avenir ».

Les conséquences sociales ne seront en rien moins dramatiques. Au contraire, la récession se prolongeant, « la langueur économique de ces dernières années (avant 1986 (ndlr)) se transformera en une anémie profonde à long terme. Tous les mécanismes de fonctionnement de l'économie seront grippés, comme ceux des entreprises, des administrations et des universités qui perdront beaucoup de leur dynamisme et de leur créativité. La société sera gouvernée par des gérontes, encombrées de cadres moyens en plein vieillissement et administrée par des bureaucrates de plus en plus âgés tandis que les jeunes y apparaîtront économiquement exploités - cotisations sociales obligent ! - , politiquement relégués et psychologiquement marginalisés ».

Finalement, les conditions d'un affrontement entre générations seront réunies, à tous les niveaux : entre actifs et inactifs, cotisants et retraités, bien portants et malades, payeurs et bénéficiaires. Le clivage sera à la fois financier, professionnel, culturel, moral, ethnique et politique. "Les jeunes seront de moins en moins nombreux à être à la fois actifs, cotisants, bien portants et payeurs ». Ils se trouveront bloqués dans leur carrière et attendront longtemps un avancement que leurs prédécesseurs obtenaient rapidement. Ils seront soumis aux valeurs dominantes qui seront celles de la vieillesse et qui conditionneront la vie culturelle, la vie politique et même morale. Le conservatisme aura 
le vent en poupe et la xénophobie s'intensifiera parce que les populations vieillies sont égocentriques et tolèrent mal l'immigration.

Les inégalités entre jeunes et vieux prendront le pas sur toutes les autres, même si les formes de l'affrontement dépendra largement de l'état même de la société : guerre frontale directe pour le partage des ressources, affrontements plus classiques à travers des grèves, des cortèges ou des occupations, ou encore formes anomiques se manifestant autant dans la marginalité écolo que dans le terrorisme.

\section{Une vision plus optimiste}

Terrible diagnostic s'il en est, qui ne peut laisser indifférent. Au grand tribunal des sociétés, le vieillissement serait à tous les coups reconnu coupable et la peine maxi- male prononcée. A l'époque de la sortie de l'ouvrage d'Alain Minc, l'auteur de ces lignes avait pourtant lui-même adopté une position radicalement opposée en déclarant que le vieillissement était une chance, non seulement individuelle (vivre plus longtemps et en meilleure santé) mais aussi collective (avoir une structure de population par âge plus en accord avec les transformations de l'emploi et avec la nouvelle révolution industrielle et l'entrée dans la civilisation des loisirs et des biens immatériels) ${ }^{4}$. Ce faisant, il rompait radicalement avec le modèle pessimiste anti-vieillissement qui avait dominé le monde scientifique et politique depuis de nombreuses décennies et proposait en lieu et place un nouveau modèle plus optimiste où les avantages l'emporteraient sur les inconvénients et surtout un nouveau paradigme méthodologique consistant à poser comme principe fondateur qu'il était absurde, inefficace et dangereux de vouloir transformer les structures démographiques pour les rendre compatibles avec l'environnement sociétal et que la seule attitude réaliste était de faire l'inverse, c'est-à-dire veiller à transformer nos structures économiques et politiques, ainsi que nos modes de fonctionnement collectif pour les mettre en harmonie avec les transformations de nos structures de population par âge et sexe, qui restent des éléments essentiels du fondement de nos sociétés et en conditionnent le bon ou le mauvais fonctionnement (en cas d'incompatibilités majeures).

Le pari était audacieux, mais avec le recul du temps, on peut observer que des déplacements de "supporters » se sont opérés et que les thèses alarmistes ont perdu une partie de leurs adhérents, sans avoir été pour autant évacuées de l'arène des débats politiques. Conscient de l'ampleur des changements qu'impliquent cette nouvelle approche, et des difficiles adaptations à mettre en œuvre, l'auteur qualifia cette mutation conceptuelle de révolution grise, en référence à l'intelligence (d'où l'allusion à la matière grise) dont il faudrait faire preuve pour réussir ce renversement des mentalités.

41 Le combat n'était certes pas gagné d'avance, mais des progrès non négligeables semblaient avoir été obtenus, précisément au moment où une crise majeure, non pas imprévisible (beaucoup d'observateurs l'avaient annoncée) mais sciemment camouflée, déferle à travers le monde entier, comme un tsunami financier détruisant tout sur son passage et balayant les espoirs pour des millions, sinon des milliards d'hommes et de femmes, d'une vie meilleure.

42 Mais du coup, les prédictions d'Alain Minc retrouvent une réelle actualité, puis- qu'une partie des risques qu'il dénonçait dans la décennie 80 sont sensiblement les mêmes que ceux que nous évoquions précédemment. Aurait-il eu raison avec vingt ans d'avance, confirmant ses talents de prospectiviste ou de futurologue, alors que nous avions cru 
pouvoir dénoncer à l'époque une approche archaïque, orientée vers les outils du passé plutôt que les arguments de l'avenir, et incapable de prendre une vision globale des interactions entre toutes les grandes composantes sociétales, notamment lorsque nous concluions: «A. Minc a cru décrire l'avenir et il y a seulement vu l'image déformée du passé. Sa machine à explorer le futur n'était qu'un engin à remonter le temps ! » ?5

Avions-nous tort, en étant aveuglé par les attraits de la polémique ? Indubitablement la question doit être posée sans complaisance, ni mauvaise foi. Cependant, une différence saute aux yeux dans les deux scénarios affichés, même si les conséquences annoncées sont convergentes : leur cause n'est pas identique, puisque dans la thèse défendue par $\mathrm{A}$. Minc, l'origine sans équivoque du déclenchement de la crise, c'est le vieillissement luimême qui transforme les modes de fonctionnement collectifs, réduit la production et gangrène les relations entre les générations, alors que pour nous l'évidence est que c'est la crise du système financier qui déclenche des catastrophes en chaîne et est susceptible de masquer les effets positifs du vieillissement et de renforcer ses effets négatifs. Mêmes constats à l'arrivée, mais conditions de départ différentes: là est toute la différence et elle est de taille.

Malheureusement, ceux qui ont choisi depuis longtemps leur camp et décidé une fois pour toute que le vieillissement était responsable de nombreuses nuisances collectives ne manqueront pas de tirer partir de l'opportunité actuelle qui leur est offerte de surcharger la victime expiatoire de tous les maux. Peut-être même trouveront-ils les moyens d'établir une relation de cause à effet entre le vieillissement et la crise financière, par exemple en évoquant le penchant des retraités pour des fonds de placement guidés uniquement par la recherche de rendements actuariels maximaux.

Simple querelle académique, sans grande importance, penseront certains ? Pas du tout rétorqueront d'autres, dans la mesure où la condamnation du vieillissement dans les sociétés qui en sont de plus en plus imprégnées risque d'accentuer les effets de la crise et de les éloigner de l'ère de géritude qui fondait l'espoir d'une gestion plus harmonieuse des âges.

\section{Et si on choisissait la décroissance?}

Ceci admis, toutes les issues négatives évoquées par Alain Minc seront-elles inévitables? Pas forcément, mais il est plus que probable que la crise économique entraînée par la crise financière sera à la hauteur de cette dernière, et même probablement au-delà. On en perçoit déjà les prémisses dans le fait que presque tous les pays de la zone euro sont entrés en récession, au sens précis du terme, c'est-à-dire pas seulement un ralentissement de la croissance, mais une vraie croissance négative, une première depuis la mise en place de cet accord européen sur une monnaie unique.

Paradoxalement, alors que l'idée de décroissance était devenue politiquement incorrecte, et considérée comme une terminologie exclue du vocabulaire politique, la voici qui s'installe spontanément, sans y avoir été invitée et surtout sans être voulue.

Pourtant, il aurait été judicieux d'accepter d'y réfléchir plus tôt et de la mettre en débat citoyen, tant il est vrai que les appels sans cesse réitérés à la croissance apparaissent de plus en plus comme des offrandes au veau d'or et qu'il faudra bien un jour admettre que la croissance illimitée est impossible, comme le Club de Rome ${ }^{7}$ en avait fait la brillante démonstration à des époques aujourd'hui lointaines où les crises énergétiques 
commençaient à saper les fondements de notre modèle économique d'économie libérale de marché.

La contradiction entre nos engagements écologiques et la croissance économique saute pourtant aux yeux, comme d'ailleurs celle avec la croissance démographique. La peur du déclin démographique associée à la peur de l'arrêt de l'enrichissement collectif ne sont pas théoriquement compatibles avec la lutte contre la raréfaction des ressources naturelles non renouvelables, ni avec celle contre la pollution et la destruction de l'écosystème mondial, sauf à croire que la science et la technologie seront toujours susceptibles de venir à bout des pénuries et des pollutions et de rattraper tous les équilibres compromis, un pari redoutable s'il en est.

Pour beaucoup, décroissance signifie perte de niveau de vie et retour en arrière sur l'échelle du progrès, alors qu'on devrait plutôt concevoir la décroissance comme une transformation des modes de vie, dans le sens d'une meilleure organisation, d'un moindre gaspillage et d'une plus grande frugalité. La question n'est pas de prétendre qu'il n'y aura plus de voitures mais qu'elles seront moins voraces en carburant et que leur usage sera moins impératif en raison d'une meilleure organisation de la mobilité. De même, installer de plus en plus de piscines privées dans un monde où une pénurie d'eau menace, trouverait peut-être une alternative collectivement plus intéressante dans une multiplication et une meilleure gestion des piscines publiques. Ou encore, des prix de plus en plus bas des tickets d'avion ne devraient pas être considérés comme une preuve de démocratisation du transport aérien, mais plutôt comme une incitation dangereuse à polluer davantage et à accentuer le trou d'ozone au nom d'un droit des vacanciers au dépaysement.

51 La principale raison pour laquelle les détracteurs de la décroissance ne pensent pas qu'elle puisse être mise en œuvre principalement serait l'impossibilité d'assurer une redistribution des revenus dans un contexte de raréfaction des ressources. Or, rien n'est moins sûr si des politiques fiscales adéquates sont adoptées qui visent à réduire les inégalités et à mieux répartir le produit collectif, fut-il en déclin modéré, et si parallèlement une communication est organisée qui vise à sensibiliser le public sur les enjeux de la décroissance et les valeurs novatrices qui la sous-tendent.

52 Quoiqu'il en soit, il est difficile de nier que les risques de repli identitaire sont plus importants en période crise. Chaque catégorie sociale tend à se focaliser sur ses acquis et à refuser les partages. Mais c'est aussi particulièrement vrai pour les générations qui pourraient s'arcbouter sur des positions d'isolement et de méfiance les unes par rapport aux autres. Au moment où la coopération des générations apparaît la plus indispensable, un tel repli apporterait un coup d'arrêt décisif aux tentatives actuelles de développer des solidarités intergénérationnelles. Le pire serait le démantèlement total ou partiel des systèmes de protection sociale, une hypothèse actuellement peu plausible en raison de la défiance à l'égard du système financier, mais qui pourrait se concrétiser si les engagements des pouvoirs publics réduisaient davantage leurs marges budgétaires. Par contre, une stagnation des prestations et des transferts sociaux aggraverait la situation des catégories les plus défavorisées, y compris les âgés pauvres ne bénéficiant que d'allocations de survie.

53 Car il ne fait plus guère de doute que la lutte des classes qui a accompagné tout le XXème siècle se transformera en conflits de générations durant le XXIème siècle, et même peutêtre en guerre ouverte si les conditions d'affrontement direct sont remplies, ce qui semble devoir être le cas avec le déclenchement de la crise. 

seront suffisamment fortes pour résister à la déferlante de la crise actuelle. Sinon, tous les efforts du milieu associatif et des pouvoirs publics pour améliorer les relations intergénérationnelles resteront vains, dans la mesure où le développement des microsolidarités à travers des projets de proximité dépend intrinsèquement de l'existence de macro-solidarités collectives, comme le sont les systèmes de protection sociale, qui sont là pour assurer la cohérence et l'intégration de tous les niveaux inférieurs de coopération intergénérationnelle.

Mais l'espoir ultime réside dans la présence d'une volonté collective de renouer (ou non) un contrat social intergénérationnel qui refonde les solidarités entre les générations et qui redéfinisse la place des âges dans nos sociétés, sans exclusion ni marginalisation.

\section{BIBLIOGRAPHIE}

BÖRSCH-SUPAN, A. et al., (eds.) (2005), Health Ageing and Retirement in Europe. First results from the survey of Health Ageing and Retirement in Europe (SHARE), Mannheim, Mannheim Research Institute for the Economics of Ageing (MEA).

BÖRSCH-SUPAN, A. et al., (2008), Health, Ageing and Retirement in Europe (2004-2007). Starting the Longitudinal Dimension. Mannheim, Mannheim Research Institute for the Economics of Ageing (MEA).

CAISSE NATIONALE D’ASSURANCE VIEILLESSE (CNAV) (2008), Le niveau de vie des retraités, Retraite et Société, Vol. 4, n. 56 Paris, La Documentation française.

CAISSE NATIONALE D'ASSURANCE VIEILLESSE (CNAV) (2007), Les retraites en Europe, Retraite et Société, Vol. 1, n. 50 Paris, La Documentation française.

CAISSE NATIONALE D'ASSURANCE VIEILLESSE (CNAV) (2005), Le soutien aux personnes âgées en Europe, Retraite et Société, Vol. 3, n. ${ }^{\circ} 46$ Paris, La Documentation française.

CAISSE NATIONALE D'ASSURANCE VIEILLESSE (CNAV) (2003), L'Europe du grand âge : entre familles et institutions, Retraite et Société, Vol. 1, n. ${ }^{\circ}$ 38, Paris, La Documentation française.

CHASTELAND, Jean-Claude, LORIAUX, Michel, ROUSSEL, Louis (2004), Démographie 2000. Une enquête internationale par Internet auprès des démographes, Bruxelles, Académia-Bruylant.

LATOUCHE, Serge (2006), Le Pari de la décroissance, Paris, Fayard.

LORIAUX, M., REMY, D. (eds.) (2006), La Retraite au quotidien. Modes de vie, représentations, espoirs et inquiétudes des personnes âgées, Bruxelles, De Boeck.

LORIAUX, Michel (1990), “ Il sera une fois ... la révolution grise. Jeux et enjeux autour d'une profonde mutation sociétale ", in LORIAUX, M., REMY, D. et VILQUIN, E., Populations âgées et révolution grise. Les hommes et les sociétés face à leur vieillissement, Chaire Quetelet 86, Louvain-laNeuve, Editions Ciaco, pp. 3-32.

MEADOWS, D., MEADOWS, D. (1973), Halte à la croissance ? Rapport sur les limites de la croissance, Paris, Ed Fayard.

Configurações, 4 | 2012 


\section{NOTES}

1. A. Minc, La Machine égalitaire, Paris, Grasset, 1987: « expansion et jeunesse finissent toujours par aller de pair, de même que récession et vieillissement » (p. 60).

2. Commission européenne, Livre vert : face aux changements démographiques, une nouvelle solidarité entre générations, Bruxelles, 2005. « On n'a jamais vu dans l'histoire une croissance (économique) sans berceaux» (p. 21).

3. En sauvant de la mort des nouveaux-nés et des jeunes enfants, le rétrécissement de la pyramide à sa base était forcément moins important et moins rapide.

4. M. Loriaux, "Il sera une fois ... la révolution grise. Jeux et enjeux autour d'une profonde mutation sociétale", in M. Loriaux, D. Remy et E. Vilquin, Populations âgées et révolution grise. Les hommes et les sociétés face à leur vieillissement, Chaire Quetelet 86, Louvain-la-Neuve, Editions Ciaco, 1990, pp. 3-32.

5. M. Loriaux, op. cit., p.18.

6. Voir par exemple, Serge Latouche, Le Pari de la décroissance, Paris, Fayard, 2006 : « la société de croissance n'est pas souhaitable pour au moins trois raisons : elle engendre une montée des inégalités et des injustices, elle crée un bien-être largement illusoire, elle ne suscite pas pour les " nantis " eux-mêmes une société conviviale mais une " antisociété » malade de sa richesse » (pp. 53-57)

7. D. Meadows et D. Meadows, Halte à la croissance ? Rapport sur les limites de la croissance, Ed Fayard, 1973.

\section{RÉSUMÉS}

L'auteur revient d'abord sur ce qu'il appelle «l'invention» du vieillissement, dans la mesure où, à l'origine du phénomène, les mutations structurelles démographiques n'ont pas été très perçues, ou prises en compte, l'attention s'étant polarisée autour de la dénatalité et de la dépopulation qui menaçait. Et de fait, le vieillissement démographique n'était guère visible qu'à travers les statistiques des rapports des groupes d'âge, le mot lui-même étant dû au démographe français Alfred Sauvy qui l'utilisa pour la première fois en 1929. Depuis lors, bien des changements ont eu lieu, le principal étant sans doute que la cause première, et longtemps unique, du vieillissement, à savoir la baisse de la fécondité a été détrônée par la cause seconde qui était le recul de la mortalité dont les progrès mesurés à travers l'espérance de vie bénéficient dorénavant essentiellement aux personnes âgées. Le vieillissement est donc à la fois individuel (des personnes âgées de plus en plus nombreuses qui vivent de plus en plus longtemps) et collectif (le groupe des aînés dont l'importance relative augmente sans cesse par rapport au groupe des jeunes).

De simplement démographique qu'il était à l'origine, le vieillissement est devenu sociétal, en déployant ses effets dans pratiquement tous les secteurs de la vie collective (formation, emploi, santé, protection sociale, finances publiques, etc.).

Or, c'est précisément au moment où les sociétés vieillissantes entrent dans une phase cruciale de leur évolution que survient une crise séculaire, financière et économique, d'une ampleur inégalée. Certaines de ses conséquences ont été immédiatement visibles, comme la perte du 
pouvoir d'achat d'une partie de la population dont les revenus dépendent principale- ment de l'épargne ; principalement les retraités. Pour beaucoup de personnes âgées la déconvenue a été forte en découvrant l'ampleur de leurs pertes dues au simple fait qu'elles ont fait confiance à des banquiers véreux, inconscients ou fous. Et, à l'heure présente, rien ne garantit que le système bancaire et financier international aura tiré les leçons de cette crise, bien au contraire. Une crise qui est d'ailleurs loin d'avoir épuisé tous ses effets néfastes comme en témoignent les pertes d'emploi, les fermetures d'entreprises, les délocalisations d'activité, etc.

Les vieux ne sont d'ailleurs pas les seules victimes et tous les âges sont menacés, de sorte qu'on peut s'attendre à un durcissement des relations intergénérationnelles qui pourrait dériver vers la guerre des âges si souvent évoquée. L'histoire donnerait ainsi raison à des auteurs catastrophistes, comme l'essayiste Alain Minc qui stigmatisait déjà le vieillissement dès 1987 dans son ouvrage sur la "Machine égalitaire ». L'avenir dira si ces scénarios apocalyptiques revisités sont les plus plausibles ou si les politiques des âges mises en œuvre permettront de limiter les dégâts occasionnés par la crise et de renforcer la cohésion intergénérationnelle. Sans être d'un optimisme à toute épreuve, l'auteur ne craint pas d'évoquer une piste paradoxale en avançant l'idée que la décroissance (du moins une décroissance limitée, réfléchie et programmée) pourrait être le meilleur moyen d'éviter que les digues de solidarité qui avaient été érigées durant le XXème siècle en créant la sécurité sociale pour lutter contre l'individualisme, l'égoïsme et la solitude soient suffisamment renforcées pour résister à la déferlante de la crise actuelle.

O autor começa por abordar o que designa de «invenção» do envelhecimento, uma vez que as transformações demográficas estruturais na origem do fenómeno não foram perceptíveis ou não foram consideradas, tendo a atenção sido focalizada na ameaça da redução da natalidade e decréscimo da população. Com efeito, o envelhecimento demográfico era apenas visível nas estatísticas sobre a importância relativa dos grupos etários, cabendo ao demógrafo francês Alfred Sauvy a autoria desta designação, utilizada pela primeira vez em 1929.

Desde esta data, ocorreram inúmeras transformações, sendo a principal o facto de a causa primordial do envelhecimento, durante muito tempo a única causa do fenómeno, a saber, a redução da fecundidade, ter sido destronada pela causa secundária do envelhecimento que é o recuo da mortalidade avaliado através da esperança de vida que passa a beneficiar sobretudo as pessoas idosas. $O$ envelhecimento é pois simultanea- mente individual (idosos cada vez mais numerosos e que vivem cada vez mais tempo) e colectivo (a importância relativa do grupo dos idosos não cessa de aumentar em relação ao grupo dos jovens).

Sendo na origem simplesmente demográfico, o envelhecimento tornou-se social, afectando praticamente todos os sectores da vida colectiva (formação, emprego, saúde, segurança social, finanças públicas, etc.).

Ora, precisamente no momento em que as sociedades em envelhecimento entram na fase crucial da sua evolução surge uma crise secular, financeira e económica, de uma dimensão sem igual. Algumas das suas consequências foram imediatamente visíveis como a perda do poder de compra de uma parte da população cujos rendimentos depen- diam sobretudo da poupança, como os reformados. Para muitos idosos o infortúnio foi claro quando descobriram a dimensão das suas perdas devidas simplesmente ao facto de terem confiado em banqueiros fraudulentos, inconscientes ou loucos. Presentemente, nada garante que o sistema bancário e financeiro internacional tenha retirado as lições desta crise, muito pelo contrário. Uma crise que está longe aliás de ter esgotado todos os seus efeitos nefastos como o testemunham as perdas de emprego, o encerramento de empresas, as deslocalizações de actividade, etc.

Os idosos não são as únicas vítimas, todas as idades estão ameaçadas, sendo de prever um endurecimento das relações intergeracionais que poderia derivar numa guerra de gerações, tantas vezes evocada. A História daria assim razão a autores catastróficos como o ensaísta Alain Minc que estigmatizava o envelhecimento já desde 1987 na sua obra sobre a «Máquina 
igualitária».

o futuro dirá se estes cenários apocalípticos revisitados são os mais plausíveis ou se as políticas implementadas permitirão limitar os estragos ocasionados pela crise e reforçar a coesão intergeracional. Sem ser de um optimismo a toda a prova, o autor não teme evocar uma pista paradoxal, avançando a ideia de que o crescimento negativo (pelo menos um crescimento negativo limitado, reflectido e programado) poderia ser a melhor forma de assegurar que as pontes de solidariedade que foram erigidas durante o século XX criando uma segurança social para lutar contra o individualismo, o egoísmo e a solidão sejam suficientemente reforçadas para resistir à desfraldada crise actual.

The author firstly approaches what he calls the «invention» of ageing as the structural demographic transformations in the origin of that phenomenon had not been perceived or had not been considered, since attention was focused on the reduction of birth rates and the decline of population. Demographic ageing was visible only in the statistics of age-groups and it was the French demographer, Alfred Sauvy, that first used the concept, in 1929.

Since then, countless transformations have occurred. The overthrow of the original cause of ageing fertility decline by a secondary cause mortality throw-back is the foremost example of these transformations.

Ageing must be understood as a phenomenon that is simultaneously individual (increasing number of elderly who live longer) and collective (increasing ageing rates).

Thus, what started as a demographic occurrence became a social experience with impacts on every sector of social life (education, employment, health, welfare, public finances, etc.).

Recently, just when the ageing societies were get- ting to the crucial point of their development, a major secular, financial and economical crisis burst. There were immediate consequences. For those that depended on savings, such as pensioners, purchasing power was lost. For many elderly people the misfortune was clear when the losses caused by fraudulent, non-conscientious or crazed bankers were reckoned. There are no guarantees that the international financial systems have learned their lessons from this cri- sis. Its noxious effects are far from over, as increasing unemployment, company closures, relocations and so on testify.

Elderly people are not the only victims of this financial crisis; it affects every age-group. This could drift into the harshening of intergenerational relations and a "war" of ages, so many times put forward. History would then confirm catastrophic prophecies like the one written by Alain Minc about the stigmatization of ageing in his 1987's book, “The equalitarian machine".

The future will tell if these apocalyptic scenarios are the most likely or if public policies directed to the aged will limit the impacts of the crisis and reinforce intergenerational cohesion.

Without being too optimistic, the author concludes with a paradoxical suggestion proposing that a negative population growth (at least a limited, rational and planned negative growth) can be the best way to reinforce the bonds that in the $\mathrm{XX}^{\text {th }}$ century made social policies possible (fighting individualism, selfishness and loneliness) and, therefore, to face this crisis.

INDEX

Palavras-chave : envelhecimento, crise financeira, relações intergeracionais

\section{AUTEUR}

MICHEL LORIAUX

Institut de Démographie - Université Catholique de Louvain 
claude-michel.loriaux@uclouvain.be 